\title{
Correction to: The 8-parameter Fisher-Bingham distribution on the sphere
}

\section{Tianlu Yuan ${ }^{1}$}

Published online: 30 September 2020

(c) Springer-Verlag GmbH Germany, part of Springer Nature 2020

\section{Correction to: Computational Statistics https://doi.org/10.1007/s00180-020-01023-w}

In the original publication of the article, the corrections in Eq. (13) were missed, in which $2 v-1$ was changed to $2 v$ in the exponent.

The corrected Eq. (13) is as given below:

$$
\begin{aligned}
c_{8}= & 2 \int_{0}^{\pi} \sum_{l, k, j=0}^{\infty}\left\{\frac{\kappa^{2(l+k)} \beta^{j} v_{2}^{2 l} v_{3}^{2 k}}{(2 l) !(2 k) ! j !} e^{\kappa v_{1} \cos \theta} \sin ^{2 v} \theta\right. \\
& \left.\times \sum_{i=0}^{j}(-\eta)^{i}\left(\begin{array}{c}
j \\
i
\end{array}\right) B(u-i, i+w)\right\} \mathrm{d} \theta
\end{aligned}
$$

The original article has been updated.

Publisher's Note Springer Nature remains neutral with regard to jurisdictional claims in published maps and institutional affiliations.

The original article can be found online at https://doi.org/10.1007/s00180-020-01023-w.

Tianlu Yuan

tyuan@icecube.wisc.edu

1 Department of Physics and Wisconsin IceCube Particle Astrophysics Center, University of Wisconsin, Madison, WI 53706, USA 